\title{
How to manage travel fatigue and jet lag in athletes? A systematic review of interventions
}

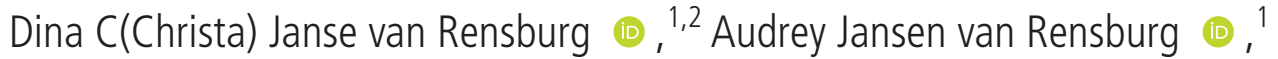 \\ Peter Fowler (1) , ${ }^{3}$ Hugh Fullagar (1) , ${ }^{4}$ David Stevens (1) , ${ }^{5}$ Shona Halson (10), \\ Amy Bender, ${ }^{7}$ Grace Vincent (i) ${ }^{8}$ Amanda Claassen-Smithers, ${ }^{9}$ Ian Dunican (i), ${ }^{10}$ \\ Gregory Daniel Roach, ${ }^{11}$ Charli Sargent, ${ }_{11}^{11}$ Michele Lastella, ${ }^{11}$ Tanita Cronje (i) ${ }^{12}$
}

- Additional material is published online only. To view please visit the journal online (http://dx.doi.org/10.1136/ bjsports-2019-101635).

For numbered affiliations see end of article.

\section{Correspondence to}

Professor Dina C(Christa) Janse van Rensburg, Faculty of Health Science, Section Sports Medicine \& Sport Exercise Medicine and Lifestyle Institute (SEMLI), University of Pretoria, Pretoria, Gauteng, South Africa; christa.jansevanrensburg@up. ac.za

Accepted 15 March 2020 Published Online First 17 April 2020

\section{Check for updates}

(c) Author(s) (or their employer(s)) 2020. No commercial re-use. See rights and permissions. Published by BMJ.

\section{To cite: Janse van} Rensburg DC(Christa), Jansen van Rensburg A, Fowler $P$, et al. Br I Sports Med 2020:54:960-968.

\section{ABSTRACT}

Objectives We investigated the management of travel fatigue and jet lag in athlete populations by evaluating studies that have applied non-pharmacological interventions (exercise, sleep, light and nutrition), and pharmacological interventions (melatonin, sedatives, stimulants, melatonin analogues, glucocorticoids and antihistamines) following long-haul transmeridian travel-based, or laboratory-based circadian system phase-shifts.

Design Systematic review

\section{Eligibility criteria}

Randomised controlled trials (RCTs), and non-RCTs including experimental studies and observational studies, exploring interventions to manage travel fatigue and jet lag involving actual travel-based or laboratory-based phase-shifts. Studies included participants who were athletes, except for interventions rendering no athlete studies, then the search was expanded to include studies on healthy populations.

Data sources Electronic searches in PubMed, MEDLINE, CINAHL, Google Scholar and SPORTDiscus from inception to March 2019. We assessed included articles for risk of bias, methodological quality, level of evidence and quality of evidence.

Results Twenty-two articles were included: 8 non-RCTs and 14 RCTs. No relevant travel fatigue papers were found. For jet lag, only 12 athlete-specific studies were available (six non-RCTs, six RCTs). In total (athletes and healthy populations), 11 non-pharmacological studies (participants 600; intervention group 290; four non-RCTs, seven RCTs) and 11 pharmacological studies (participants 1202; intervention group 870; four non-RCTs, seven RCTs) were included. For nonpharmacological interventions, seven studies across interventions related to actual travel and four to simulated travel. For pharmacological interventions, eight studies were based on actual travel and three on simulated travel.

Conclusions We found no literature pertaining to the management of travel fatigue. Evidence for the successful management of jet lag in athletes was of low quality. More field-based studies specifically on athlete populations are required with a multifaceted approach, better design and implementation to draw valid conclusions.

\section{PROSPERO registration number}

The protocol was registered in the International Prospective Register of Systematic Reviews (PROSPERO: (RD42019126852).

\section{INTRODUCTION}

Globally, athletes are frequently required to embark on long-haul transmeridian travel for competition purposes for example, Olympic Games, IAAF Diamond League Series and the Super Rugby Tournament, exposing them to travel fatigue and jet lag. ${ }^{1-6}$ Travel fatigue follows any long journey irrespective of the mode of travel and can accumulate over time. ${ }^{2-5}$ Jet lag follows travel across multiple time-zones $(>3)$ with subsequent desynchronisation of the circadian system to the new external environment at the destination. ${ }^{2}{ }^{4-6}$ The human circadian system requires time to adjust, leading to persistence of jet lag symptoms including sleep disruption, daytime fatigue, gastrointestinal disturbances and reduced performance. ${ }^{257}$ Literature on jet lag focusses mostly on interventions with a determined phase response curve, able to induce a circadian phase-shift. ${ }^{38-13}$ Few studies describe effective interventions to recover (ie, reverse a phase-shift) from jet lag.

Current clinical applications rely heavily on opinions, collective experience and findings from simulated travel (laboratory-based) studies due to a lack of well-designed actual travel (field-based) studies. ${ }^{1}$ The most promising interventions have been tested in a simulated travel environment in the laboratory, but not necessarily in an actual travel scenario. ${ }^{3}{ }^{14}$ Even though simulated travel and actual travel are not directly comparable, laboratory-based studies provide valuable information that can be used to design field-based studies. ${ }^{15}$ The latter is expensive, difficult to control and population recruitment challenging especially in the athlete context. Due to these constraints, most of the literature comes from non-athlete studies. ${ }^{114} 1617$

For this paper we systematically reviewed nonpharmacological interventions (exercise, sleep, light (exposure/avoidance), nutrition (mealtime/ composition)), and pharmacological interventions (melatonin, sedatives, stimulants, melatonin analogues, glucocorticoids and antihistamines), that can be applied in managing travel fatigue and jet lag in athlete populations following transmeridian travel.

\section{METHODOLOGY}

This review was conducted according to the Preferred Reporting Items for Systematic Review and Meta-Analysis (PRISMA) statement. ${ }^{18}$ 


\section{Data sources and search strategy}

We conducted a comprehensive literature search from inception to March 2019 to obtain relevant peer-reviewed publications. Initially, we studied all relevant literature in relation to the management of travel fatigue and/or jet lag. We included all populations (animal and human) to identify any intervention used. We then selected only those interventions that were research-based and relevant to our review question regarding athlete populations. We excluded interventions based on opinions and collective experience. Relevance was determined based on the collective expertise and experience of the authors, all of which are active in this specific area of research. Electronic databases searched included PubMed, MEDLINE, CINAHL, Google Scholar and SPORTDiscus. Authors were assigned to project groups covering a specific non-pharmacological intervention (ie, exercise, sleep, light (exposure/avoidance), nutrition (mealtime/ composition), and pharmacological intervention (ie, melatonin, sedatives, stimulants, melatonin analogues, glucocorticoids and antihistamines). To exclude the risk of inter-individual variability in screening and identification of studies, project groups consisted of different authors (overlapping group membership), and two authors (CJvR, AJvR) independently performed literature searches on all interventions. A search strategy was developed using relevant Medical Subject Heading search terms, adding identified subject keywords in different combinations. Boolean operators 'OR' and 'AND' were used to combine or exclude keywords in the search, resulting in more focussed and refined results (provided in online supplementary file 1). We manually searched the reference list of included studies to identify other relevant studies. Some of the interventions did not yield any athlete-specific studies, but showed promise in healthy population studies. After intense discussions and careful consideration, the author group decided to, where available, include only athlete-specific studies for interventions (ie, exercise, sleep, light, melatonin and sedatives) but to infer from healthy population studies for those interventions (ie, nutrition, stimulants and melsatonin analogues) where no athlete-specific studies were found. No year limits were applied. We conducted a search in July 2019 and before submission to identify any new eligible studies.

\section{Eligibility criteria}

Inclusion criteria for selected studies:

1. Randomised control trials (RCTs) and non-randomised control trials (non-RCTs) including experimental studies and observational studies (cross-sectional, case-control and cohort studies) exploring an intervention to manage travel fatigue or jet lag.

2. Involve long-haul transmeridian travel-based, or laboratorybased circadian system phase-shifts (ie, studies that observed usefulness of interventions following a phase-shift of the circadian system).

3. Published as original research in a peer-reviewed journal.

4. Published as full-text.

5. Athlete specific (healthy individual studies were included only for those interventions where no athlete specific studies were available).

6. Participants $\geq 18$ years of age.

7. No restrictions towards gender, ethnicity and follow-up duration (in case of longitudinal studies).

8. Only human participants.

9. Only English language.

Exclusion criteria for selected studies:
1. If light-dark or sleep-wake cycle was shifted without a period of travel or simulated travel (ie, studies that observed efficacy of interventions to induce a phase-shift in the circadian system).

2. Investigating shift work disorder and social jet lag.

3. Participants with mobility impairment (eg, Paralympians), diseased populations (eg, cancer) or animals.

4. Reviews, case series, case reports, editorials and abstracts.

\section{Study selection}

We identified relevant studies on each intervention combining findings from each project group and the independent searches performed (CJvR, AJvR). From the compiled list, the titles and abstracts for each article was screened to identify relevant studies. Full-texts of potentially relevant articles were reviewed to determine eligibility for the systematic review. All authors agreed on inclusion of studies. In the event of any disagreement, accord of decision was made by three authors (CJvR, AJvR, TC), when at least two reviewers independently agreed on the eligibility of the studies, and achieved consensus on which studies to include and which data to extract.

\section{Data extraction}

For each specific intervention, data were extracted from eligible studies. Data included level of evidence, publication details (first author, study title, year of publication), study design (type of study), study duration, participants (sex, sample size, age), control group or not, induced phase-shift, aim of the study, intervention used, measurements used and main outcomes.

\section{Risk of bias, methodological quality, level of evidence and quality of evidence}

We evaluated RCT studies using the Cochrane Risk of Bias Tool. ${ }^{19}$ The domains of risk of bias assessment included: selection bias, reporting bias, performance bias, detection bias and attrition bias. For non-RCTs, we used the Downs and Black Tool to assess each study in its entirety, not simply a specific outcome or result. ${ }^{2021}$

Additionally, the level of evidence of each eligible study was critically appraised using the Oxford Centre for Evidence-based Medicine (OCEBM) levels of evidence. ${ }^{22}$ The OCEBM ranking levels evaluate studies according to best evidence for clinical focus (diagnosis, prognosis, harm, treatment and prevention) based on the relative strengths and weaknesses of each study design. We used the clinical focus for 'treatment and prevention'.

The quality of the evidence of each intervention was assessed with the Grading of Recommendations, Assessment, Development and Evaluation (GRADE) system. ${ }^{23}$ Criteria included risk of bias, directness, consistency, precision and publication bias. Each outcome is graded as either high, moderate, low or very low, and reflects the degree of confidence in the effect estimate. Three authors (CJvR, AJvR, TC) assessed all studies independently for bias, methodological quality, level of evidence and quality of evidence, with any disagreements resolved by consensus from other authors.

\section{RESULTS}

\section{Study identification}

A PRISMA flow chart documents the search and selection process for non-pharmacological interventions (figure 1A) and pharmacological interventions (figure 1B). The initial electronic database search returned 1366 non-pharmacological and 5622 pharmacological articles. Additional articles were sourced from 


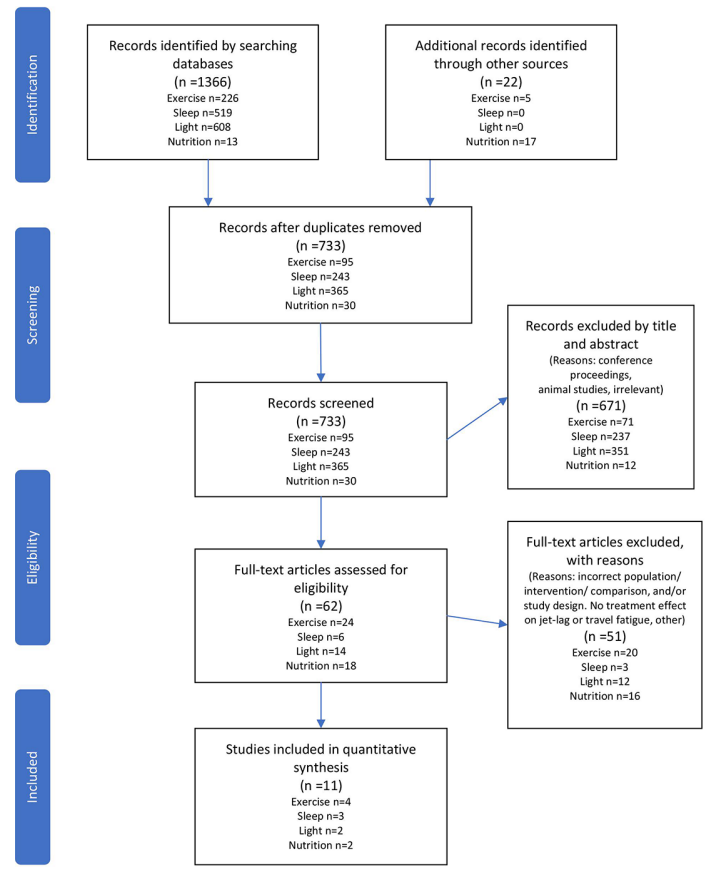

A

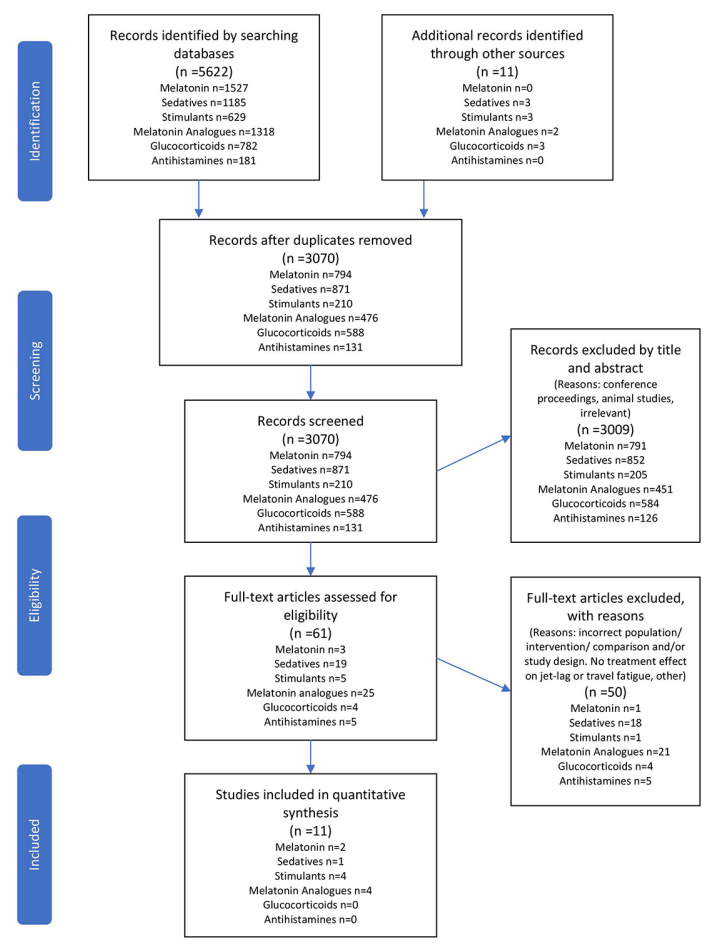

B

Figure 1 Preferred Reporting Items for Systematic Review and Meta-Analysis (PRISMA) flow diagram of the systematic review (A) on nonpharmacological interventions for the management of travel fatigue and jet lag in athletes and (B) on pharmacological interventions for the management of travel fatigue and jet lag in athletes.

the reference lists of the reviewed articles. Duplicate records were removed. After reviewing titles and abstracts, and reviewing the full-text of potential articles, 11 articles remained for each intervention category. Two reviewers (CJvR and AJvR) re-evaluated all relevant articles for each intervention.

\section{Data synthesis and analysis}

We grouped and summarised studies as non-pharmacological interventions (exercise, sleep, light (exposure/avoidance), nutrition (mealtime/composition)) and pharmacological interventions (melatonin, sedatives, stimulants, melatonin analogues, glucocorticoids and antihistamines); as depicted in online supplementary file 2 . After broad consultation with statisticians and investigating the literature regarding the guidelines on conducting a meta-analysis, a collective decision was made that a meta-analysis was not feasible. This was due to the diverse nature and lack of consistency of the studies regarding population, flight direction, study design (implementation of the intervention protocol differed), outcomes measured and statistical parameters. A sensible combination of such diversity was not possible and could produce misleading results. ${ }^{24-28}$

\section{Non-pharmacological interventions}

No relevant travel fatigue papers were found. For jet lag, three interventions (exercise: $4,{ }^{29-32}$ sleep: $3,{ }^{33-35}$ light: $2^{3036}$ ) yielded athlete-specific studies. There were no athlete-specific studies for the category on nutrition (mealtime/composition) and we subsequently inferred from two healthy population studies. ${ }^{37} 38$ Seven studies across interventions related to actual travel (exercise: $2{ }^{29}{ }^{30}$ sleep: $1,{ }^{35}$ light: $2,^{30}{ }^{36}$ nutrition: $2^{37}{ }^{38}$ ) and four to simulated travel (exercise: $2,^{31} 32$ sleep: $2^{33}{ }^{34}$ ). In total 11 non-pharmacological intervention studies (participants 600; intervention group 290) were included, of which four were nonRCTs $^{293037}$ and seven were RCTs ${ }^{31-3638}$ (table 1A,B).

\section{Pharmacological interventions}

We did not find any relevant papers on travel fatigue. For jet lag, limited athlete-specific studies were available (melatonin: 2,3039 sedatives: $1^{40}$ ). For the remainder of the interventions the search was expanded to include studies on healthy populations (stimulants: $4,{ }^{41-44}$ melatonin analogues: $4^{45-48}$ ). In total eight studies were based on actual travel (melatonin: $2,^{30}{ }^{39}$ sedatives: $1,{ }^{40}$ stimulants: $4,{ }^{41-44}$ melatonin analogues: $1^{48}$ ) and three on simulated travel (melatonin analogues: $3^{45-47}$ ). We found no eligible studies for the categories on glucocorticoids and antihistamines. Overall, 11 pharmacological intervention studies (participants 1202; intervention group 870) were included, of which four were non-RCTs ${ }^{30394045}$ and seven were RCTs ${ }^{41-4446-48}$ (table 2A,B).

Risk of bias, methodological quality and quality of evidence Details of the risk of bias and methodological quality assessment are provided in online supplementary file 3 .

\section{Non-pharmacological interventions}

The degree to which RCT studies met the risk of bias criteria varied from moderate-to-high (table 1A). The methodological quality of the non-RCTs were poor (table $1 \mathrm{~B}$ ). The quality of evidence of studies demonstrated no serious problems with directness, but some to major problems with consistency, precision and publication bias (table $1 \mathrm{~A}, \mathrm{~B})$.

\section{Pharmacological interventions}

The degree to which RCTs met the risk of bias criteria varied from low-to-moderate (table 2A). The methodological quality 
Table 1 Grading of evidence for non-pharmacological interventions to manage jet lag in athletes

\begin{tabular}{|c|c|c|c|c|c|c|c|c|c|c|}
\hline \multicolumn{11}{|c|}{ A. Randomised controlled trials } \\
\hline Category & $\begin{array}{l}\text { Number of } \\
\text { studies }\end{array}$ & $\begin{array}{l}\text { Total participants } \\
\text { (intervention } \\
\text { group) }\end{array}$ & $\begin{array}{l}\text { Actual }(A) \text { or } \\
\text { simulated }(S) \\
\text { travel }\end{array}$ & $\begin{array}{l}\text { Athlete } \\
\text { specific }\end{array}$ & Risk of bias & Consistency & Directness & Precision & $\begin{array}{l}\text { Publication } \\
\text { bias }\end{array}$ & $\begin{array}{l}\text { Total } \\
\text { GRADE rating }\end{array}$ \\
\hline Exercise & \multirow[t]{2}{*}{2} & $35(18)$ & & & \multirow[t]{2}{*}{ High } & * & $t$ & $\ddagger$ & \multirow[t]{2}{*}{ *§ } & \multirow{2}{*}{$\begin{array}{l}\text { Very low } \\
+ \\
\text { serious RoB } \\
\text { downgraded one } \\
\text { level from low }\end{array}$} \\
\hline $\begin{array}{l}\text { Barger, et a } \beta^{31} \\
\text { Yamanaka, et }\left.a\right|^{32}\end{array}$ & & $\begin{array}{l}18(9) \\
17(9)\end{array}$ & $\begin{array}{l}S \\
S\end{array}$ & $\begin{array}{l}\text { Yes } \\
\text { Yes }\end{array}$ & & $\begin{array}{l}\text { Clinical } \\
\text { homogeneity, } \\
\text { methodological } \\
\text { heterogeneity }\end{array}$ & $\begin{array}{l}\text { (The evidence } \\
\text { answers } \\
\text { the review } \\
\text { question) }\end{array}$ & $\begin{array}{l}\text { (Small sample } \\
\text { sizes, no effect } \\
\text { sizes reported, } \\
\text { randomisation } \\
\text { process unclear) }\end{array}$ & & \\
\hline Sleep & \multirow[t]{2}{*}{3} & $48(40)$ & & & \multirow[t]{2}{*}{ High } & ${ }^{*} / \ddagger$ & $\dagger$ & $\ddagger$ & \multirow[t]{2}{*}{ *§ } & \multirow[b]{2}{*}{$\begin{array}{l}\text { Low } \\
++ \\
\text { serious RoB } \\
\text { downgraded } \\
\text { one level from } \\
\text { moderate }\end{array}$} \\
\hline $\begin{array}{l}\text { Petit, et al }{ }^{\beta 3}(2014) \\
\text { Petit, et al }{ }^{34}(2018) \\
\text { Straub, et } a l^{35}\end{array}$ & & $\begin{array}{l}16(16) \\
16(16) \\
16(8)\end{array}$ & $\begin{array}{l}S \\
S \\
A\end{array}$ & $\begin{array}{l}\text { Yes } \\
\text { Yes } \\
\text { Yes }\end{array}$ & & $\begin{array}{l}\text { Only two of three } \\
\text { studies used same } \\
\text { methodology and } \\
\text { intervention }\end{array}$ & $\begin{array}{l}\text { (The evidence } \\
\text { answers } \\
\text { the review } \\
\text { question) }\end{array}$ & $\begin{array}{l}\text { (Small sample sizes, } \\
\text { no effect sizes } \\
\text { reported) }\end{array}$ & & \\
\hline Light & \multirow[t]{2}{*}{1} & \multirow[t]{2}{*}{$22(11)$} & & & \multirow[t]{2}{*}{ Moderate } & $\ddagger$ & $t$ & $* / \ddagger$ & \multirow[t]{2}{*}{ *§ } & \multirow{2}{*}{$\begin{array}{l}\text { Low } \\
++\end{array}$} \\
\hline Thompson, et al ${ }^{36}$ & & & A & Yes & & $\begin{array}{l}\text { Participants } \\
\text { females only }\end{array}$ & $\begin{array}{l}\text { (The evidence } \\
\text { answers } \\
\text { the review } \\
\text { question) }\end{array}$ & $\begin{array}{l}\text { (Effect size } \\
\text { reported, small } \\
\text { sample size) }\end{array}$ & & \\
\hline $\begin{array}{l}\text { Nutrition (meal timing/ } \\
\text { composition) }\end{array}$ & \multirow[t]{2}{*}{1} & \multirow[t]{2}{*}{$61(31)$} & & & \multirow[t]{2}{*}{ High } & $\ddagger$ & $\dagger$ & $\ddagger$ & \multirow[t]{2}{*}{$\ddagger$} & \multirow[t]{2}{*}{$\begin{array}{l}\text { Very low } \\
+\end{array}$} \\
\hline Ruscitto and Ogden ${ }^{38}$ & & & $A$ & No & & $\begin{array}{l}\text { Many } \\
\text { confounders, } \\
\text { many limitations }\end{array}$ & $\begin{array}{l}\text { (The evidence } \\
\text { answers } \\
\text { the review } \\
\text { question) }\end{array}$ & $\begin{array}{l}\text { (Low response rate, } \\
\text { self-reported) }\end{array}$ & & \\
\hline TOTAL & 7 & $166(100)$ & & & & & & & & \\
\hline $\begin{array}{l}\text { *Some problems. } \\
\text { †No serious problems. } \\
\text { ‡Major problems. } \\
\text { §All athlete specific studies }\end{array}$ & & & & & & & & & & \\
\hline
\end{tabular}

\begin{tabular}{|c|c|c|c|c|c|c|c|c|c|c|}
\hline \multicolumn{11}{|c|}{ B. Non-Randomised controlled trials } \\
\hline Category & $\begin{array}{l}\text { Number of } \\
\text { studies }\end{array}$ & $\begin{array}{l}\text { Total participants } \\
\text { (intervention } \\
\text { group) }\end{array}$ & $\begin{array}{l}\text { Actual }(A) \text { or } \\
\text { simulated (S) } \\
\text { travel }\end{array}$ & $\begin{array}{l}\text { Athlete } \\
\text { specific }\end{array}$ & $\begin{array}{l}\text { Methodological } \\
\text { quality }\end{array}$ & Consistency & Directness & Precision & $\begin{array}{l}\text { Publication } \\
\text { bias }\end{array}$ & $\begin{array}{l}\text { Total } \\
\text { GRADE rating }\end{array}$ \\
\hline Exercise & 2 & $40(34)$ & & & Poor & $* / \ddagger$ & $t$ & $\ddagger$ & *§ & Very low \\
\hline $\begin{array}{l}\text { Montaruli, et } a l^{29} \\
\text { Cardinali, et }\left.a\right|^{30}\end{array}$ & & $\begin{array}{l}18(12) \\
22(22)\end{array}$ & $\begin{array}{l}A \\
A\end{array}$ & $\begin{array}{l}\text { Yes } \\
\text { Yes }\end{array}$ & $\begin{array}{l}10 / 28 \\
10 / 28\end{array}$ & $\begin{array}{l}\text { One study } \\
\text { no control } \\
\text { group, clinical } \\
\text { heterogeneity, } \\
\text { methodological } \\
\text { heterogeneity }\end{array}$ & $\begin{array}{l}\text { (The evidence } \\
\text { answers } \\
\text { the review } \\
\text { question) }\end{array}$ & $\begin{array}{l}\text { (Small sample sizes, } \\
\text { no effect sizes } \\
\text { reported) }\end{array}$ & & + \\
\hline Sleep & 0 & & & & & & & & & \\
\hline Light & 1 & $22(22)$ & & & Poor & $\ddagger$ & $t$ & $* / \ddagger$ & *§ & Very low \\
\hline Cardinali, et $\left.a\right|^{30}$ & & & A & Yes & $10 / 28$ & $\begin{array}{l}\text { Participants } \\
\text { males only, } \\
\text { multi factorial } \\
\text { interventions }\end{array}$ & $\begin{array}{l}\text { (The evidence } \\
\text { answers } \\
\text { the review } \\
\text { question) }\end{array}$ & $\begin{array}{l}\text { (Small sample sizes, } \\
\text { no effect sizes } \\
\text { reported) }\end{array}$ & & + \\
\hline $\begin{array}{l}\text { Nutrition (meal timing/ } \\
\text { composition) }\end{array}$ & 1 & $372(134)$ & & & Poor & $\ddagger$ & $t$ & $\ddagger$ & $\ddagger$ & $\begin{array}{l}\text { Very low } \\
+\end{array}$ \\
\hline $\begin{array}{l}\text { Reynolds and } \\
\text { Montgomery }\end{array}$ & & $186 \times 2(95+39)$ & $A$ & No & $10 / 28$ & $\begin{array}{l}\text { Participants could } \\
\text { choose }\end{array}$ & $\begin{array}{l}\text { (The evidence } \\
\text { answers } \\
\text { the review } \\
\text { question) }\end{array}$ & $\begin{array}{l}\text { (No effect sizes, } \\
\text { changing sample } \\
\text { size) }\end{array}$ & & \\
\hline TOTAL & 4 & $434(190)$ & & & & & & & & \\
\hline
\end{tabular}

*Some problems.

tNo serious problems

¥Major problems

$\S$ All athlete-specific studies have small sample sizes that may lead to publication bias.

GRADE, Grading of Recommendations, Assessment, Development and Evaluation; non-RCTs, non-randomised control trials; RoB, risk of bias.

of the non-RCTs varied from poor-to-moderate (table 2B). The quality of evidence of studies demonstrated no serious problems with directness, a variety of problems with consistency, some to major problems with precision and some problems with publication bias (table 2A,B).

\section{Outcomes of interventions}

Non-pharmacological interventions

- Exercise: Four athlete-specific studies (two non-RCT ${ }^{29} 30$ and two $\mathrm{RCT}^{3132}$ ) recognised exercise as an intervention. All studies (two laboratory-based ${ }^{31} 32$ and two field-based ${ }^{29}{ }^{30}$ ) suggested that exercise may help with resynchronisation of the circadian system following long-haul travel, but that timing of the exercise session was important. Conclusion: Judiciously timed exercise may help to recover from jet lag. (non-RCT: GRADE $\oplus$ OOO; RCT: GRADE $\oplus$ OOO)

- Sleep: Napping (two laboratory-based studies ${ }^{33}$ 34) and diaphragmatic breathing combined with listening to a sleep training CD (one field-based study ${ }^{35}$ ) were explored in three athlete-specific RCTs. Napping had some positive influence 
Table 2 Grading of evidence pharmacological interventions to manage jet lag in athletes

\begin{tabular}{|c|c|c|c|c|c|c|c|c|c|c|}
\hline \multicolumn{10}{|c|}{ A. Randomised controlled trials } & \multirow[b]{2}{*}{$\begin{array}{l}\text { Total } \\
\text { GRADE } \\
\text { rating }\end{array}$} \\
\hline Category & $\begin{array}{l}\text { Number of } \\
\text { studies }\end{array}$ & $\begin{array}{l}\text { Total participants } \\
\text { (intervention } \\
\text { group) }\end{array}$ & $\begin{array}{l}\text { Actual }(A) \text { or } \\
\text { simulated (S) } \\
\text { travel }\end{array}$ & $\begin{array}{l}\text { Athlete } \\
\text { specific }\end{array}$ & Risk of bias & Consistency & Directness & Precision & Publication bias & \\
\hline Melatonin & 0 & & & & & & & & & \\
\hline Sedatives & 0 & & & & & & & & & \\
\hline Stimulants & 4 & $508(339)$ & & & Low-to-moderate & $* / \dagger$ & $t$ & */キ & $* / \dagger$ & $\begin{array}{l}\text { Moderate-to- } \\
\text { high }\end{array}$ \\
\hline $\begin{array}{l}\text { Rosenberg, et a }\left.\right|^{41} \\
\text { Lagarde, et } a l^{42} \\
\text { Piérard, et } a l^{43} \\
\text { Beaumont, et } a l^{44}\end{array}$ & & $\begin{array}{l}427(285) \\
27(18) \\
27(18) \\
27(18)\end{array}$ & $\begin{array}{l}A \\
A \\
A \\
A\end{array}$ & $\begin{array}{l}\text { No } \\
\text { No } \\
\text { No } \\
\text { No }\end{array}$ & & $\begin{array}{l}\text { One study } \\
\text { explored } \\
\text { armodafinil, } \\
\text { the other three } \\
\text { studies had } \\
\text { clinical and } \\
\text { methodological } \\
\text { homogeneity }\end{array}$ & $\begin{array}{l}\text { (The evidence } \\
\text { answers } \\
\text { the review } \\
\text { question) }\end{array}$ & $\begin{array}{l}\text { Effect size and } \\
\text { Cls not reported }\end{array}$ & $\begin{array}{l}\text { Pharma } \\
\text { industry driven } \\
\text { (armodafinil) }\end{array}$ & +++ \\
\hline Melatonin analogues & 3 & $635(480)$ & & & Low-to-moderate & $\dagger$ & $\dagger$ & * & * & Moderate \\
\hline $\begin{array}{l}\text { Rajaratnam, et } a l^{46} \\
\text { Richardson, et a }\left.\right|^{47} \\
\text { Zee, et } a l^{48}\end{array}$ & & $\begin{array}{l}39+411(31+308) \\
75(60) \\
110(81)\end{array}$ & $\begin{array}{l}S \\
S \\
A\end{array}$ & $\begin{array}{l}\text { No } \\
\text { No } \\
\text { No }\end{array}$ & & $\begin{array}{l}\text { Well designed } \\
\text { and executed } \\
\text { studies }\end{array}$ & $\begin{array}{l}\text { (The evidence } \\
\text { answers } \\
\text { the review } \\
\text { question) }\end{array}$ & & $\begin{array}{l}\text { Pharma industry } \\
\text { driven }\end{array}$ & +++ \\
\hline Glucocorticoids & 0 & & & & & & & & & \\
\hline Antihistamines & 0 & & & & & & & & & \\
\hline TOTAL & 7 & $1143(819)$ & & & & & & & & \\
\hline
\end{tabular}

B. Non-Randomised controlled trials

\begin{tabular}{|c|c|c|c|c|c|c|c|c|c|c|}
\hline Category & $\begin{array}{l}\text { Number of } \\
\text { studies }\end{array}$ & $\begin{array}{l}\text { Total participants } \\
\text { (intervention } \\
\text { group) }\end{array}$ & $\begin{array}{l}\text { Actual (A) or } \\
\text { simulated (S) } \\
\text { travel }\end{array}$ & $\begin{array}{l}\text { Athlete } \\
\text { specific }\end{array}$ & $\begin{array}{l}\text { Methodological } \\
\text { quality }\end{array}$ & Consistency & Directness & Precision & Publication bias & $\begin{array}{l}\text { Total } \\
\text { GRADE } \\
\text { rating }\end{array}$ \\
\hline Melatonin & 2 & $34(34)$ & & & Poor & $\ddagger$ & $\dagger$ & $\ddagger$ & *§ & Very low \\
\hline $\begin{array}{l}\text { Cardinali, et a }\left.\right|^{30} \\
\text { Manfredini, et } a l^{39}\end{array}$ & & $\begin{array}{l}22(22) \\
12(12)\end{array}$ & $\begin{array}{l}\mathrm{A} \\
\mathrm{A}\end{array}$ & $\begin{array}{l}\text { Yes } \\
\text { Yes }\end{array}$ & $\begin{array}{l}10 / 28 \\
9 / 28\end{array}$ & $\begin{array}{l}\text { Both studies } \\
\text { no control } \\
\text { group, clinical } \\
\text { heterogeneity, } \\
\text { methodological } \\
\text { heterogeneity }\end{array}$ & $\begin{array}{l}\text { (The evidence } \\
\text { answers } \\
\text { the review } \\
\text { question) }\end{array}$ & $\begin{array}{l}\text { Effect size } \\
\text { andCls not } \\
\text { reported, no } \\
\text { control group }\end{array}$ & & + \\
\hline Sedatives & 1 & $17(9)$ & & & Poor & $\ddagger$ & $t$ & $\ddagger$ & ${ }^{*} \S$ & \\
\hline Reilly, et al ${ }^{40}$ & & $17(9)$ & A & $\begin{array}{l}\text { Mixed } \\
\text { (gymnasts } \\
\text { and support } \\
\text { staff in one } \\
\text { group) }\end{array}$ & $9 / 28$ & $\begin{array}{l}\text { Mixed gymnasts } \\
\text { and support staff } \\
\text { into one group }\end{array}$ & $\begin{array}{l}\text { (The evidence } \\
\text { answers } \\
\text { the review } \\
\text { question) }\end{array}$ & $\begin{array}{l}\text { Mixed athletes } \\
\text { and non- } \\
\text { athletes }\end{array}$ & & $\begin{array}{l}\text { Very low } \\
+\end{array}$ \\
\hline
\end{tabular}

\begin{tabular}{|c|c|c|c|c|c|c|c|c|c|c|}
\hline Stimulants & 0 & & & & & & & & & \\
\hline Melatonin analogues & 1 & $8(8)$ & & & Moderate & $* / \dagger$ & $\dagger$ & $\ddagger$ & * & Low \\
\hline Nickelsen, et a $\left.\right|^{45}$ & & & $S$ & No & $19 / 28$ & $\begin{array}{l}\text { Although small } \\
\text { sample size, } \\
\text { well described } \\
\text { methodology and } \\
\text { reported results }\end{array}$ & $\begin{array}{l}\text { (The evidence } \\
\text { answers } \\
\text { the review } \\
\text { question) }\end{array}$ & $\begin{array}{l}\text { Effect size } \\
\text { andCls not } \\
\text { reported }\end{array}$ & & ++ \\
\hline
\end{tabular}

\begin{tabular}{lll} 
Glucocorticoids & 0 & \\
\hline Antihistamines & 0 & \\
TOTAL & 4 & $59(51)$ \\
\hline
\end{tabular}

*.Some problems

tNo serious problems.

¥Major problems

$\S$ All athlete specific studies have small sample sizes that may lead to publication bias.

GRADE, Grading of Recommendations, Assessment, Development and Evaluation; RCTs, randomised controlled trials; RoB, risk of bias.

on reducing jet lag, but different nap durations or schedules were not tested. The combined intervention ${ }^{35}$ of breathing and sleep training had no positive outcome. Conclusion: Current evidence from athlete-specific laboratory-based and field-based studies does not support the use of sleep interventions for reducing jet lag symptoms, but more specific research is required. (RCT: GRADE $\oplus \oplus \mathrm{OO}$ )

- Light: Two field-based athlete-specific studies (one non$\mathrm{RCT}^{30}$ and one $\mathrm{RCT}^{36}$ ) investigated the effect of light on recovery from jet lag. The non- $\mathrm{RCT}^{30}$ reported faster resynchronisation after light treatment, but was part of a multifactorial intervention also including judiciously timed exercise and melatonin administration, making inferences for a specific intervention difficult. The $\mathrm{RCT}^{36}$ specifically observed the effect of a light intervention for a small group of female athletes and they reported no benefit of light treatment. Conclusion: Athlete-specific studies with light as intervention to reduce jet lag shows contrasting results. (non-RCT: GRADE $\oplus$ OOO; RCT: GRADE $\oplus \oplus \mathrm{OO}$ )

- Nutrition: (mealtime/composition): No athlete-specific studies were identified. The search was then expanded to include healthy populations. A non- $\mathrm{RCT}^{37}$ and a $\mathrm{RCT}^{38}$ 
reported that mealtime may alleviate jet lag following actual travel. The non- $\mathrm{RCT}^{37}$ further implied that the meal composition can have an effect. However, both studies had many confounders. Conclusion: Appropriate timing and composition of meals may be a valuable treatment strategy to reduce jet lag, but athlete-specific research is required. (non-RCT: GRADE $\oplus$ OOO; RCT: GRADE $\oplus$ OOO)

\section{Pharmacological interventions}

- Melatonin: Two field-based non-RCTs ${ }^{30} 39$ explored exogenous melatonin administration as an intervention in athletes. One study ${ }^{30}$ claimed that melatonin administration reduced time for resynchronisation of the circadian system following long-haul travel, the other study ${ }^{39}$ had mixed results. Neither study had a control group and sample sizes were small. One study had multiple interventions ${ }^{30}$ (melatonin, exercise and light exposure). Conclusion: Exogenous melatonin may be important to recover from jet lag, but larger studies with control groups are required. (non-RCT: GRADE $\oplus$ OOO)

- Sedatives: Only one non-RCT ${ }^{40}$ involved an athlete population and investigated benzodiazepines (temazepam) as a possible intervention following actual travel. In addition, the intervention group was not homogeneous and included both athletes and support staff. No athlete-specific studies on short-acting hypnotics (eg, zolpidem, zopiclone) are available. Conclusion: Current evidence on the use of sedatives in the athlete population is limited. (non-RCT: GRADE $\oplus$ OOO)

- Stimulants: No athlete-specific studies are available. Healthy population studies yielded four well-designed field-based RCTs. ${ }^{41-44}$ One large study ${ }^{41}$ specifically examined armodafinil and reported promising results as treatment strategy for alleviation of jet lag. Three smaller studies, ${ }^{42-44}$ each using the same methodology and participants reported improved physical performance, resynchronisation of hormonal rhythms and better sleep after timely ingestion of caffeine. Conclusion: Although athlete-specific literature is not available, studies in healthy populations show encouraging results to reduce jet lag symptoms and improve resynchronisation of the circadian system following long-haul travel. (RCT: GRADE $\oplus \oplus \oplus \mathrm{O})$

- Melatonin analogues: We found no athlete-specific studies. The search was then expanded to include studies on healthy populations. Three well-designed RCTs (two laboratorybased $^{4647}$ and one field-based ${ }^{48}$ ) were identified investigating tasimelteon (a phase II and phase III study reported in one publication ${ }^{46}$ ) and ramelteon. ${ }^{47} 48$ One laboratorybased non-RCT ${ }^{45}$ investigated LY15673. Improvement of jet lag symptoms was reported for all analogues, but was dose and time dependent. Conclusion: Correct administration of a melatonin analogue may be a valuable treatment strategy to reduce jet lag, but athlete-specific and field-based research is required. (non-RCT: GRADE $\oplus \oplus \mathrm{OO}$; RCT: GRADE $\oplus \oplus \oplus \mathrm{O})$

\section{DISCUSSION}

Our systematic review of all available literature found no evidence for non-pharmacological or pharmacological interventions to manage travel fatigue in athletes. When present, the evidence for interventions to manage jet lag in athletes was of poor quality.

\section{Exercise}

Some evidence from laboratory-based studies suggests that exercise may induce phase-shifts comparable in size to bright light ${ }^{49}$ and the timing of exercise appears to be critical. A phase response curve to a 1-hour bout of moderate-intensity exercise was recently established. ${ }^{50}$ We identified four studies reporting the positive effect of judiciously timed exercise on adaptation of the circadian system to a new time-zone following a transmeridian flight. ${ }^{29-32}$ Only two studies were field-based, involving actual air travel and athletes or trained participants. ${ }^{29}{ }^{30}$ Montaruli $e{ } \mathrm{l}^{29}$ reported that the timing of moderate-intensity exercise prior to a 9-hour flight west, across six time-zones from Milan to compete in the New York Marathon, had a significant impact on sleep and adaptation of the circadian system posttravel. ${ }^{29}$ Specifically, evening training (19:00 to $\left.21: 00\right) 5$ days prior to the flight resulted in improved sleep quality on night 1 and 2 post-flight, compared with morning training (07:00 to 09:00) and no training. Evening training also resulted in a greater phase-delay (4-hour) in the circadian system compared with morning training (1-hour) and control (1-hour). No significant differences in subjective jet lag symptoms existed between the groups. Unexplored differences between the groups, particularly light exposure could confound results. The inclusion of a longer post-flight monitoring period and a more reliable marker of circadian phase such as core body temperature or melatonin as opposed to actigraphy would have been more insightful. Cardinali $e t a l^{30}$ exposed a group of professional male soccer players travelling 12-hours west from Buenos Aires to Tokyo to a combined intervention of $3 \mathrm{mg}$ melatonin, sunlight exposure/ avoidance and physical exercise exposure/avoidance to facilitate circadian adaptation. ${ }^{30}$ These soccer players had a significantly shorter resynchronisation period $(2.13 \pm 0.88$ days) rather than the expected 6 days after a 12-hours time-zone change. This study was limited by being observational and was poorly controlled with exercise intensity appearing to be self-selected by participants. Additionally, being a combined intervention, it is difficult to ascertain the contribution of exercise to the circadian adaptation.

While there is no evidence that the effects of light and exercise are additive, appropriate times to conduct exercise for its phase-shifting properties appear to coincide with that of light exposure. ${ }^{51}$ Judiciously timed outdoor training sessions could be used by coaches and athletes as a strategy to reduce severity and duration of jet lag symptoms. Our review returned studies that used vastly different exercise protocols and doses. The effects of physical activity on circadian phase and on jet lag symptoms require further exploration.

\section{Sleep}

Literature supports sleep preservation as an important strategy of jet lag management. ${ }^{8}$ In an athlete specific study, Petit et $a l^{34}$ showed that a strategic $20 \mathrm{~min}$ nap between 08:00 and 09:00 following a simulated 5-hour phase-shift, may alleviate jet lag and result in increased cognitive performance. Petit $e{ }{ }^{33}$ further conducted a randomised crossover counterbalanced design investigating a simulated 5-hour phase-advance, and found no changes in physical performance or facilitation of resynchronisation after a $20 \mathrm{~min}$ post-lunch nap. Athletes, however, did experience an increase in the time to fall asleep on the night after the nap. ${ }^{33}$ Novel strategies are often employed to support sleep during travel or to manage jet lag. One study investigated the effect of a combined intervention (diaphragmatic breathing and listening to a sleep training CD) to improve sleep, mood and 
performance, and to reduce jet lag after a rapid time-zone shift of 10-hours east (Sweden to Australia). This resulted in no significant findings for any measure of sleep, mood or performance. ${ }^{35}$

No studies have evaluated the impact of sleep timing during travel on the succeeding sleep-wake cycle at the destination ${ }^{52}$ and there are no athlete-specific studies on sleep hygiene. Sleep hygiene has been shown to help healthy participants recover from travel fatigue following 24-hours of simulated international travel and although good sleep hygiene may not force a phase-shift of the circadian system, bad sleep habits including incidental exposure to electronic equipment may induce phaseshifts, even in the wrong direction. ${ }^{53}$ Research on the sleep patterns of athletes during and following transmeridian travel is limited and needs further investigation. ${ }^{54-56}$

\section{Light}

Appropriately timed light exposure/avoidance to facilitate re-entrainment of the circadian system is widely recommended. ${ }^{77-59}$ To facilitate maximum circadian phase-shift, the duration, intensity and wavelength applied are most critical. ${ }^{12} 6061$ Observing the effect of light treatment in athletes, a RCT of 22 elite female soccer players assessed the efficacy of light intervention to facilitate recovery after a 5 -hour and 8-hour phase-advance following air travel. ${ }^{36}$ Results indicated insignificant and variable effects of light on physical functioning, diet, gastrointestinal and sleep symptoms. As discussed in the exercise intervention section Cardinali $e t a l^{30}$ reported that a multifactorial intervention (exercise, light and melatonin) significantly reduced the period ( $2.13 \pm 0.88$ days) to resynchronise as opposed to the expected 6 days after a 12-hours time-zone change. Due to the multifactorial nature of the interventions, the direct impact of light exposure is difficult to elucidate. The lack of evidence for the effective use of light may also be attributed to small sample sizes and interindividual variability in circadian phase. Although appropriate light exposure/avoidance proved effective in laboratory-based studies, field-based studies are difficult to implement due to the level of control required for example, avoiding incidental light exposure and practicalities such as sharing of light boxes and timing of exercise sessions. ${ }^{36}$ Future research should focus on a multifaceted approach.

\section{Nutrition}

When manipulating jet lag, the master clock (the conductor situated in the suprachiasmatic nucleus) rather than the peripheral clocks (situated in most cells of all tissues) are targeted. Peripheral clocks may not respond to the same interventions that influence the master clock, such as light application, and resynchronisation may be different to that of the master clock. ${ }^{8}$ Presumably food will affect peripheral clocks, and nutrition may be a promising intervention to enhance circadian system adjustment. ${ }^{62}{ }^{63}$ Human studies are limited, and we could only find two, non-athlete studies in healthy individuals, that studied the effect of nutrition on circadian phase-shifts. ${ }^{3738}$ In a case control study soldiers were given the option of using the Argonne diet 4 days before deployment and on returning home (USA to South Korea). Those using the Argonne diet reported fewer symptoms of jet lag. ${ }^{37}$ The Argonne diet consists of 4 days of alternating between feasting (no calorie limit: a protein-rich breakfast and lunch, with a carbohydrate-rich supper) and fasting $(<800$ $\mathrm{kcal} / \mathrm{day}$ : fruit for breakfast and lunch, and vegetable soup for supper). The soldiers' interpretation of and adherence to the diet was not supervised and jet lag symptoms were based on self-report. Restricted feeding or fasting to adjust the body clock may not be appropriate for athletes. The value of fasting remains to be tested and weighed against the potential disadvantage of compromising current, proven sports nutrition recommendations for optimising training, competition performance and recovery. ${ }^{64}$ In a RCT, long-haul cabin crew implemented a self-selected meal plan, primarily focussed on eating three regular meals, synchronised with the light dark cycle, on arrival. These cabin crew reported fewer symptoms of jet lag. ${ }^{38}$ This dietary strategy aligns well with current, proven sports nutrition recommendations and could be a worthwhile consideration for athletes.

We could not find any human or athlete-specific studies evaluating the effects of hydration during transmeridian travel. Although research on dietary interventions is in its infancy, such interventions are usually easy to implement, have fewer potential side effects, are relatively cheap and more acceptable to athletes. ${ }^{14}$

\section{Melatonin}

A previous Cochrane Review reported melatonin to be effective and safe in adult travellers. ${ }^{16}$ Currently, there is little or no evidence supporting the ingestion of exogenous melatonin to accelerate resynchronisation in athletes. We found two single-group field-based studies, both with small sample sizes and no control groups. ${ }^{3039}$ Cardinali $e t ~ a l^{30}$ reported a significantly shorter period $(2.13 \pm 0.88$ days $)$ of resynchronisation in contrast to the expected 6 days after a 12-hours time-zone change using combined interventions ( $3 \mathrm{mg}$ melatonin, sunlight exposure/avoidance and physical exercise exposure/avoidance). Manfredini $e t a l^{39}$ reported widely differing effects of melatonin ingestion on the circadian system. Although laboratory studies have showed that melatonin can induce a phase-shift, ${ }^{65} 66$ a study in a healthy population that travelled east across 10 time-zones indicated that $5 \mathrm{mg}$ of melatonin ingested at local bedtime had neither a chronobiotic nor a hypnotic effect. ${ }^{67}$

Phase-shifting effects of light can be partially counteracted by appropriately timed exogenous melatonin, ${ }^{68}$ and an additive effect on phase-shift can occur when correctly timed. ${ }^{69}$ There is however limited evidence that exogenous melatonin can enhance resynchronisation of the circadian system in athletes following travel. ${ }^{81567}$ Purity and dose accuracy are often not regulated and several countries have usage restrictions. ${ }^{70}$

\section{Sedatives}

We could only find one athlete-specific case-control study, including gymnasts and support staff who ingested benzodiazepines. ${ }^{40}$ Participants in the intervention group reported fewer jet lag symptoms and improved mean sleep quality from day 1 to day 5. Most studies investigating the use of sedatives to mitigate jet lag and travel fatigue in healthy populations only managed to present low quality evidence. ${ }^{70-74}$ Limited research shows that benzodiazepines can accelerate re-entrainment of the circadian system (chronobiotic effect) and normalise markers of sleep-wake homeostasis (hypnotic effect). ${ }^{71}$ Midazolam has been shown to significantly lengthen sleep after eastward travel. ${ }^{72}$ Zopiclone has been shown to accelerate readjustment of the rest/activity rhythm and normalise the phase relationship between sleep and the temperature rhythm after westward travel, ${ }^{73}$ as well as increase sleep duration and quality. ${ }^{74}$ Sedatives may offer an alternative solution to alleviating jet lag symptoms by inducing sleep on arrival to normalise circadian rhythms to the arrival time-zone. Strictly speaking, sedatives reduce jet lag symptoms by inducing sleep rather than via a chronobiotic 
effect. ${ }^{40} 75-77$ Sedatives may have side effects including amnesia, confusion $^{78}$ and immobility which, in restricted spaces such as aircraft, increase the risk of deep vein thrombosis. ${ }^{79}$ Given the conflicting evidence for the efficacy of sedatives, coupled with the risk of abuse and long-term harm, we are hesitant to recommend sedatives for regular jet lag management.

\section{Stimulants}

In this review, we could only find studies investigating the effects of the stimulants, armodafinil and caffeine to manage jet lag, in healthy non-athletes. For armodafinil, a double-blind RCT revealed increased alertness after $150 \mathrm{mg}$ /day following 6-hours of eastbound travel in travellers with a history of jet lag. ${ }^{41}$ In a between-subject study, caffeine, depressed cortisol release, increased alertness and enhanced physical performance. Lagarde et $a l^{42}$ found that participants who ingested $300 \mathrm{mg}$ slow-release caffeine in the local morning for 5 days, after 7-hours of eastward travel, had stronger static grip-strength performance but no improvement in dynamic jumping tasks when compared with a control group. In two other RCTs, US Military Air Force reservists who ingested $300 \mathrm{mg}$ of slow-release caffeine had lower cortisol concentrations ${ }^{43}$ and improved alertness, but poorer sleep quality. ${ }^{44}$ These results suggest that caffeine may have undesirable effects on athletes. Although caffeine is generally not seen as a chronobiotic and is used mainly to improve alertness at the destination time-zone, a recent study demonstrated a phasedelay in the circadian system following caffeine consumption. ${ }^{80}$ The topic of stimulants is contentious due to most being performance enhancing. ${ }^{81}$ Further research is required to determine appropriate timing and dose and implication of usage in athlete populations.

\section{Melatonin analogues}

Melatonin analogues were initially developed to treat sleep disorders not related to jet lag. We could not find any studies specifically on athletes, and included studies on healthy populations. In a large phase II and III double-blind RCT, ${ }^{46}$ where 450 healthy volunteers were subjected to a 5-hour phase advance, results showed that tasimelteon initiated a phase-shift as measured by melatonin onset, as well as improved subjective and objective sleep variables. Tasimelteon also seems to have the ability to treat transient insomnia associated with jet lag. ${ }^{46}$ Another double-blind $\mathrm{RCT}^{48}$ (110 healthy volunteers) investigated the effects of different dosages of ramelteon on improving sleep onset problems associated with jet lag, after crossing five time-zones east. Only the $1 \mathrm{mg}$ dose, not the $4 \mathrm{mg}$ and $8 \mathrm{mg}$, reduced the time to sleep onset. The $4 \mathrm{mg}$ dose improved daytime alertness, concentration, quality of sleep and ease of awakening. All dosages however, caused a significant reduction in the immediate memory recall test, and no significant phase-shift. ${ }^{48}$ Conflicting results were reported in a double-blind $\mathrm{RCT}^{47}$ (75 heathy volunteers) on ramelteon where the 1, 2 and $4 \mathrm{mg}$ dosages significantly advanced the phase of circadian rhythm following a 5-hour simulated phase-advance with no improvement in sleep. ${ }^{47}$ In a small (eight healthy volunteers) double-blind non$\mathrm{RCT}^{45} 5 \mathrm{mg}$ of LY156735 significantly shifted the circadian system and also enhanced daytime performance after a 9-hour simulated phase-advance. It seems as if melatonin analogues have chronobiotic effects in a healthy population when tested in a simulated environment, however more research is needed to clarify the efficacy in actual jet lag in athlete populations.

\section{Glucocorticoids and antihistamines}

No relevant studies on either glucocorticoids or antihistamines were found. Given the fact that athletes may consume these products to overcome jet lag, available information on the topic is discussed. Endogenous glucocorticoids (produced by the hypothalamus-pituitary-adrenal axis), are tightly linked with circadian rhythms, ${ }^{82}$ and are believed to prepare the body for physical activity. Exogenous glucocorticoids are used to treat a range of conditions, especially those causing systemic inflammatory responses. ${ }^{83}$ Although not investigating phase-shifts, one study in humans ${ }^{84}$ found that glucocorticoids effectively realigned circadian rhythms after 40-hours of sleep deprivation. Given the potential side effects and being listed as a banned substance, ${ }^{81}$ we do not recommend the use of glucocorticoids for overcoming jet lag in travelling athletes until more large-scale RTCs have been conducted.

Antihistamines counter the action of histamine, a compound in the body that causes itching and flushing of the skin such as in allergic and inflammatory reactions. First-generation antihistamines are able to cross the blood-brain barrier (BBB) causing sedation, with drowsiness listed as a potential side effect. ${ }^{85}$ Second-generation and third-generation antihistamines are not effective for sleep as they are unable to pass through the BBB. While effective at inducing sleep, no study has examined the efficacy or effectiveness of using antihistamines for overcoming jet lag, making recommendations difficult. Most people quickly develop a tolerance to the sedative/sleep inducing effects of antihistamines. ${ }^{8687}$ Therefore we do not recommend the use of first-generation antihistamines for overcoming jet lag or travel fatigue.

\section{Tolerability and side effect profile of interventions}

As part of management, users should know about the tolerability and possible adverse events of any intervention. Athletes in particular need to adhere to the most recent World Anti-Doping Agency (WADA) ${ }^{81}$ regulations for all pharmacological interventions. The efficacy and side effects of interventions may vary for different users, who may also experience jet lag differently. Most of the articles that we reviewed did not mention possible side effects except in clinical trial studies ${ }^{45-48}$ and few studies eluded to acceptability of the intervention to the participants. To complicate matters, athletes are usually reticent to change or add to their routines for research purposes.

\section{Strengths and limitations of this review}

This is the first systematic review on the management strategies for travel fatigue and jet lag in athletes. Athletes may differ from the average healthy population due to higher fitness and motivation levels, potentially safeguarding them from travel demands. All athlete-specific studies had small sample sizes and represented different sporting codes (eg, marathon runners, soccer players, swimmers), complicating the interpretation of results. Vast differences in methodological design, subject demographics (age and sex) and outcome measures existed within and between laboratory-based and field-based studies, confounding comparison. Most studies included a varied range of subjective (eg, sleep diaries, subjective questionnaires, visual analogue scale) and objective (eg, actigraphy, polysomnograms, grip-strength) outcome measures further complicating comparison. Studies also used different methods to determine the circadian phase, and subsequent timing of interventions. Some studies used dim light melatonin onset, others used habitual sleep time, core body temperature minimum, plasma melatonin or urinary melatonin metabolite 6-sulphatoxymelatonin as demonstrated in online supplementary file 2 . Future studies should aim to standardise outcome measures and circadian phase determination 
What is already known

- Misalignment of the circadian system with the new local time may impair cognitive and physical performance.

- Light and melatonin can induce a phase-shift in the circadian system if appropriately timed.

- Measuring the phase of the circadian system and proper timing of interventions are challenging in real life such as for sports people.

- Interventions seem to be successful in laboratory settings, but limited data are available in actual travel conditions.

\section{What are the new findings}

- Few studies investigated interventions to advance or delay circadian phase-shifts in athletes who experience jet lag.

- There is no research-based evidence to manage travel fatigue in athletes!

- Low quality evidence exists for effective interventions to recover from jet lag in athlete populations.

- Comparing studies is complicated by large intra-study variation in determining circadian phase, along with a wide variety of outcome measures.

for meaningful comparisons between studies and to enable conduction of a meta-analysis. A strength of our study is that we followed a systematic approach and although we could not perform a meta-analysis we made use of the well-validated GRADE system.

\section{CONCLUSION}

Well-designed studies assessing interventions for managing travel fatigue and jet lag in athletes in actual travel scenarios are limited. We systematically reviewed the literature and found no evidence related to travel fatigue. We could only find low quality evidence of successful interventions for managing jet lag in athletes. Some interventions only included healthy participants (nutrition, stimulants and melatonin analogues) and require testing in athlete populations. Current recommendations for managing jet lag appear to be based on opinions, collective experience and results from laboratory-based studies on nonathletes. Comparison of laboratory-based and field-based studies remains questionable with difficulties in controlling incidental light exposure and determining circadian phase in the field. We included two non-pharmacological interventions (exercise and sleep) where both laboratory-based and field-based interventions were reported. For both interventions the laboratory-based and field-based studies showed similar outcomes, but the laboratorybased studies were limited in their design and these findings should be interpreted with caution. Symptoms of travel fatigue and jet lag are entwined and focussing only on circadian re-alignment following transmeridian travel may not be sufficient to safeguard well-being. Future studies should focus on multifaceted strategies to overcome these challenges and aim to find a balance between the efficacy and practicality of interventions in well-designed field-based studies using athlete populations.

\section{Author affiliations}

${ }^{1}$ Faculty of Health Science, Section Sports Medicine \& Sport Exercise Medicine and Lifestyle Institute (SEMLI), University of Pretoria, Pretoria, Gauteng, South Africa ${ }^{2}$ Medical Board Member, International Netball Federation, Manchester, UK
${ }^{3}$ School of Exercise and Nutrition Sciences, Queensland University of Technology, Brisbane, Queensland, Australia

${ }^{4}$ Sport and Exercise Science, Faculty of Health, University of Technology Sydney, Sydney, New South Wales, Australia

${ }^{5}$ Adelaide Institute for Sleep Health, College of Medicine and Public Health, Flinders University, Adelaide, South Australia, Australia

${ }^{6}$ School of Behavioural and Health Sciences, McAuley at Banyo, Australian Catholic University, Brisbane, Queensland, Australia

${ }^{7}$ Faculty of Kinesiology, University of Calgary, Calgary, Alberta, Canada

${ }^{8}$ Appleton Institute for Behavioural Science, Central Queensland University,

Rockhampton, Queensland, Australia

${ }^{9}$ The South African Institute for Drug-Free Sport, Cape Town, South Africa

${ }^{10}$ Centre for Sleep Science, The University of Western Australia Faculty of Health and Medical Sciences, Perth, Western Australia, Australia

${ }^{11}$ Appleton Institute for Behavioural Science, Central Queensland University, Adelaide, Queensland, Australia

${ }^{12}$ Faculty of Natural and Agricultural Sciences, Department of Statistics, University of Pretoria, Pretoria, South Africa

Twitter Dina C(Christa) Janse van Rensburg @ChristaJVR, Audrey Jansen van Rensburg@Audrey_JvR, Peter Fowler @fowlerp85, Hugh Fullagar @HughFullagar, David Stevens @sleepyDJS, Shona Halson @ShonaHalson, Amy Bender @sleep4sport, Grace Vincent @PhDsleepy, Ian Dunican @sleep4perform, Gregory Daniel Roach@CBTmin, Charli Sargent @ThePhaseShifter, Michele Lastella @sleeppsyc and Tanita Cronje @Tcnita

Acknowledgements The authors would like to express their gratitude to Mrs Brenda Weder for editing, Mrs Madeleen Scheepers for upload of publications to Endnote library and to Dr Cheryl Tosh for grammar and language editing.

Contributors CJvR: Responsible for the overall idea for this review article, content concept, manuscript planning, search strategy, search and manuscript (first draft), manuscript editing. AJvR: Manuscript (first draft), content contribution, manuscript planning, search strategy, manuscript editing. All authors and co-authors searched library bases, identified relevant studies, reviewed studies, selected eligible studies, wrote the article with contributing input, manuscript editing. All authors revised and approved the final draft.

Funding The authors have not declared a specific grant for this research from any funding agency in the public, commercial or not-for-profit sectors.

Competing interests None declared.

Patient consent for publication Not required.

Provenance and peer review Not commissioned; externally peer reviewed.

\section{ORCID iDs}

Dina C(Christa) Janse van Rensburg http://orcid.org/0000-0003-1058-6992

Audrey Jansen van Rensburg http://orcid.org/0000-0003-1749-5073

Peter Fowler http://orcid.org/0000-0002-5853-9119

Hugh Fullagar http://orcid.org/0000-0001-9426-5373

David Stevens http://orcid.org/0000-0002-8412-2202

Shona Halson http://orcid.org/0000-0002-1047-3878

Grace Vincent http://orcid.org/0000-0002-7036-7823

Ian Dunican http://orcid.org/0000-0002-4000-8213

Tanita Cronje http://orcid.org/0000-0002-8861-4466

\section{REFERENCES}

1 Janse van Rensburg DC, Jansen van Rensburg A, Schwellnus MP. Coping with jet lag and protecting athlete health when travelling. Aspetar Sports Med J 2019:8:214-22 https://www.aspetar.com/journal/viewarticle.aspx?id=474

2 Samuels $\mathrm{CH}$. Jet lag and travel fatigue: a comprehensive management plan for sport medicine physicians and high-performance support teams. Clin I Sport Med 2012;22:268-73.

3 Arendt J. Managing jet lag: some of the problems and possible new solutions. Sleep Med Rev 2009;13:249-56.

4 Reilly T, Waterhouse J, Edwards B. Some chronobiological and physiological problems associated with long-distance journeys. Travel Med Infect Dis 2009;7:88-101.

5 Waterhouse J, Reilly T, Atkinson G, et al. Jet lag: trends and coping strategies. The Lancet 2007;369:1117-29.

6 Reilly T, Atkinson G, Edwards B, et al. Coping with jet-lag: a position statement for the European College of sport science. Eur J Sport Sci 2007;7:1-7.

7 Eastman Cl, Burgess HJ. How to travel the world without jet lag. Sleep Med Clin 2009;4:241-55.

8 Arendt J. Approaches to the pharmacological management of jet lag. Drugs 2018;78:1419-31.

9 Zee PC. Science of circadian rhythms. An Issue of Sleep Medicine Clinics: Elsevier Health Sciences 2015 
10 Revell VL, Molina TA, Eastman Cl. Human phase response curve to intermittent blue light using a commercially available device. J Physiol 2012;590:4859-68.

11 St Hilaire MA, Gooley JJ, Khalsa SBS, et al. Human phase response curve to a $1 \mathrm{H}$ pulse of bright white light. J Physiol 2012;590:3035-45.

12 Rüger M, St Hilaire MA, Brainard GC, et al. Human phase response curve to a single 6.5 H pulse of short-wavelength light. J Physiol 2013;591:353-63.

13 Burgess HJ, Revell VL, Molina TA, et al. Human Phase Response Curves to Three Days of Daily Melatonin: $0.5 \mathrm{mg}$ Versus $3.0 \mathrm{mg}$. J Clin Endocrinol Metab 2010;95:3325-31.

14 Bin YS, Postnova S, Cistulli PA. What works for jetlag? A systematic review of nonpharmacological interventions. Sleep Med Rev 2019;43:47-59.

15 Atkinson G, Batterham AM, Dowdall N, et al. From animal cage to aircraft cabin: an overview of evidence translation in jet lag research. Eur J Appl Physiol 2014;114:2459-68.

16 Herxheimer A, Petrie KJ, Cochrane Common Mental Disorders Group. Melatonin for the prevention and treatment of jet lag. Cochrane Database Syst Rev 2002;30.

17 Youngstedt SD, Kline CE, Elliott JA, et al. Circadian phase-shifting effects of bright light, exercise, and bright light+ exercise. J Circadian Rhythms 2016;14.

18 Liberati A, Altman DG, Tetzlaff J, et al. The PRISMA statement for reporting systematic reviews and meta-analyses of studies that evaluate healthcare interventions: explanation and elaboration. BMJ 2009:339:b2700-94.

19 Higgins JPT, Altman DG, Gotzsche PC, et al. The Cochrane collaboration's tool for assessing risk of bias in randomised trials. BMJ 2011;343:d5928.

20 Page MJ, McKenzie JE, Higgins JPT. Tools for assessing risk of reporting biases in studies and syntheses of studies: a systematic review. BMJ Open 2018;8:e019703.

21 Downs SH, Black N. The feasibility of creating a checklist for the assessment of the methodological quality both of randomised and non-randomised studies of health care interventions. J Epidemiol Community Health 1998;52:377-84.

22 Howick J, Chalmers I, Glasziou P, et al. Explanation of the 2011 Oxford centre for evidence-based medicine (OCEBM) levels of evidence (background document), 2011. Oxford centre for evidence-based medicine. Available: http://www. cebm. net/index. aspx

23 Ryan R, Hill S. How to grade the quality of the evidence. Cochrane consumers and communication group 2016.

24 Higgins JP, Green S. Cochrane Handbook for systematic reviews of interventions. John Wiley \& Sons, 2011.

25 Berstock JR, Whitehouse MR. How to prepare and manage a systematic review and meta-analysis of clinical studies. EFORT Open Reviews 2019;4:213-20.

26 Ahn E, Kang H. Introduction to systematic review and meta-analysis. Korean J Anesthesiol 2018;71:103-12.

27 Onerup A, Arvidsson D, Blomqvist Åse, et al. Physical activity on prescription in accordance with the Swedish model increases physical activity: a systematic review. $\mathrm{Br}$ J Sports Med 2019;53:383-8.

28 Luque-Suarez A, Martinez-Calderon J, Falla D. Role of kinesiophobia on pain, disability and quality of life in people suffering from chronic musculoskeletal pain: a systematic review. Br J Sports Med 2019:53:554-9.

29 Montaruli A, Roveda E, Calogiuri G, et al. The sportsman readjustment after transcontinental flight: a study on marathon runners. J Sports Med Phys Fitness 2009; $49: 372$

30 Cardinali DP, Bortman GP, Liotta G, et al. A multifactorial approach employing melatonin to accelerate resynchronization of sleep-wake cycle after a 12 time-zone westerly transmeridian flight in elite soccer athletes. J Pineal Res 2002;32:41-6.

31 Barger LK, Wright KP, Hughes RJ, et al. Daily exercise facilitates phase delays of circadian melatonin rhythm in very dim light. Am J Physiol Regul Integr Comp Physiol 2004;286:R1077-84

32 Yamanaka Y, Hashimoto S, Tanahashi Y, et al. Physical exercise accelerates reentrainment of human sleep-wake cycle but not of plasma melatonin rhythm to 8-h phase-advanced sleep schedule. Am J Physiol Regul Integr Comp Physiol 2010;298:R681-91.

33 Petit E, Mougin F, Bourdin H, et al. A 20-min nap in athletes changes subsequent sleep architecture but does not alter physical performances after normal sleep or 5-h phase-advance conditions. Eur J Appl Physiol 2014;114:305-15.

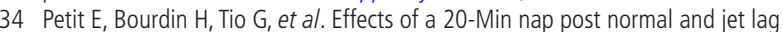
conditions on p300 components in athletes. Int I Sports Med 2018;39:508-16.

35 Straub WF, Spino MP. T-tests and confidence intervals in literature the effects of diaphragmatic breathing and sleep training on sleep, jet lag and swimming performance. Sport J 2003.

36 Thompson A, Batterham AM, Jones $\mathrm{H}$, et al. The practicality and effectiveness of supplementary bright light for reducing jet-lag in elite female athletes. Int I Sports Med 2013:34:582-9.

37 Reynolds NC, Montgomery R. Using the Argonne diet in jet lag prevention: deployment of troops across nine time zones. Mil Med 2002;167:451-3.

38 Ruscitto C, Ogden J. The impact of an implementation intention to improve mealtimes and reduce jet lag in long-haul cabin crew. Psychol Health 2017:32:61-77.

39 Manfredini R, Manfredini F, Conconi F. Standard melatonin intake and circadian rhythms of elite athletes after a transmeridian flight. J Int Med Res $2000 ; 28: 182-6$
40 Reilly T, Atkinson G, Budgett R. Effect of low-dose temazepam on physiological variables and performance tests following a westerly flight across five time zones. Int J Sports Med 2001;22:166-74.

41 Rosenberg RP, Bogan RK, Tiller JM, et al, eds. A phase 3, double-blind, randomized, placebo-controlled study of armodafinil for excessive sleepiness associated with jet lag disorder. Mayo Clin Proc. Elsevier, 2010.

42 Lagarde D, Chappuis B, Billaud PF, et al. Evaluation of pharmacological AIDS on physical performance after a transmeridian flight. Med Sci Sports Exerc 2001;33:628-34

43 Piérard C, Beaumont M, Enslen M, et al. Resynchronization of hormonal rhythms after an eastbound flight in humans: effects of slow-release caffeine and melatonin. Eur $J$ Appl Physiol 2001;85:144-50.

44 Beaumont M, Batéjat D, Piérard C, et al. Caffeine or melatonin effects on sleep and sleepiness after rapid eastward transmeridian travel. J Appl Physio/ 2004;96:50-8.

45 Nickelsen T, Samel A, Vejvoda M, et al. Chronobiotic effects of the melatonin agonis LY 156735 following a simulated $9 \mathrm{H}$ time shift: results of a placebo-controlled trial. Chronobiol Int 2002:19:915-36.

46 Rajaratnam SMW, Polymeropoulos MH, Fisher DM, et al. Melatonin agonist tasimelteon (VEC-162) for transient insomnia after sleep-time shift: two randomised controlled multicentre trials. The Lancet 2009;373:482-91.

47 Richardson GS, Zee PC, Wang-Weigand S, et al. Circadian phase-shifting effects of repeated ramelteon administration in healthy adults. J Clin Sleep Med 2008;4:456-61.

48 Zee PC, Wang-Weigand S, Wright KP, et al. Effects of ramelteon on insomnia symptoms induced by rapid, eastward travel. Sleep Med 2010;11:525-33.

49 Eastman C, Hoese EK, Youngstedt SD. Phase-shifting human circadian rhythms with exercise during the night shift. Physiol Behav 1995;58:1287-91.

50 Youngstedt SD, Elliott JA, Kripke DF. Human circadian phase-response curves for exercise. J Physiol 2019;597:2253-68.

51 Roach GD, Sargent C. Interventions to minimize jet lag after westward and eastward flight. Front Physiol 2019;10.

52 Duffield RFP, Crowcroft S, Mendham A, et al. Team sport performance following eastward and westward long-haul air travel. conference: annual Congress of the European College of sport science. Malmö, Sweden 2015 https://www.researchgate. net/publication/279868580_Team_Sport_Performance_Following_Eastward_and_ Westward_Long-haul_Air_Travel/comments

53 Fowler PM, Duffield R, Morrow I, et al. Effects of sleep hygiene and artificial bright light interventions on recovery from simulated international air travel. Eur J Appl Physiol 2015:115:541-53.

54 Waterhouse Jet al. Identifying some determinants of "jet lag" and its symptoms: a study of athletes and other travellers. Br J Sports Med 2002;36:54-60.

55 Reilly T, Edwards B. Altered sleep-wake cycles and physical performance in athletes. Physiol Behav 2007;90:274-84.

56 Lee A, Galvez JC. Jet lag in athletes. Sports Health 2012;4:211-6.

57 Sack RL. The pathophysiology of jet lag. Travel Med Infect Dis 2009;7:102-10.

58 Khalsa SBS, Jewett ME, Cajochen C, et al. A phase response curve to single bright light pulses in human subjects. J Physiol 2003;549:945-52.

59 Czeisler C, Kronauer R, Allan J, et al. Bright light induction of strong (type 0 ) resetting of the human circadian pacemaker. Science 1989;244:1328-33.

60 Rimmer DW, Boivin DB, Shanahan TL, et al. Dynamic resetting of the human circadian pacemaker by intermittent bright light. Am J Physiol Regul Integr Comp Physiol 2000;279:R1574-9.

61 Boivin DB, Duffy JF, Kronauer RE, et al. Dose-Response relationships for resetting of human circadian clock by light. Nature 1996;379:540-2.

62 Oike H. Modulation of circadian clocks by nutrients and food factors. Biosci Biotechnol Biochem 2017;81:863-70.

63 Potter GDM, Cade JE, Grant PJ, et al. Nutrition and the circadian system. Br J Nutr 2016;116:434-42.

64 Thomas D, Erdman K, Burke L. American College of sports medicine joint position statement. nutrition and athletic performance. Med Sci Sports Exerc 2016;48:543-68.

65 Rajaratnam SMW, Dijk D-J, Middleton B, et al. Melatonin phase-shifts human circadian rhythms with no evidence of changes in the duration of endogenous melatonin secretion or the 24-hour production of reproductive hormones. J Clin Endocrinol Metab 2003;88:4303-9.

66 Arendt J, Skene DJ. Melatonin as a chronobiotic. Sleep Med Rev 2005:9:25-39.10.1016/j.smrv.2004.05.002

67 Edwards BJ, Atkinson G, Waterhouse J, et al. Use of melatonin in recovery from jet-lag following an eastward flight across 10 time-zones. Ergonomics 2000;43:1501-13.

68 Deacon S, Arendt J. Adapting to phase shifts, II. Effects of melatonin and conflicting light treatment. Physiol Behav 1996;59:675-82.

69 Revell VL, Burgess HJ, Gazda CJ, et al. Advancing human circadian rhythms with afternoon melatonin and morning intermittent bright light. J Clin Endocrinol Metab 2006;91:54-9

70 Forbes-Robertson S, Dudley E, Vadgama P, et al. Circadian disruption and remedial interventions. Sports Medicine 2012:42:185-208.

71 Buxton OM, Copinschi G, Van Onderbergen A, et al. A benzodiazepine hypnotic facilitates adaptation of circadian rhythms and sleep-wake homeostasis to an eight hour delay shift simulating westward jet lag. Sleep 2000;23:1-13. 
72 Lavie P. Effects of midazolam on sleep disturbances associated with westward and eastward flights: evidence for directional effects. Psychopharmacology 1990;101:250-4.

73 Daurat A, Benoit O, Buguet A. Effects of zopiclone on the rest/activity rhythm after a westward flight across five time zones. Psychopharmacology 2000;149:241-5.

74 Jamieson AO, Zammit GK, Rosenberg RS, et al. Zolpidem reduces the sleep disturbance of jet lag. Sleep Med 2001;2:423-30.

75 Ambesh P, Shetty V, Ambesh S, et al. Jet lag: Heuristics and therapeutics. J Family Med Prim Care 2018:7:507.

76 Donaldson E, Kennaway DJ. Effects of temazepam on sleep, performance, and rhythmic 6-sulphatoxymelatonin and cortisol excretion after transmeridian travel. Aviation, space, and environmental medicine 1991.

77 Hirschfeld U, Moreno-Reyes R, Akseki E, et al. Progressive elevation of plasma thyrotropin during adaptation to simulated jet lag: effects of treatment with bright light or zolpidem. J Clin Endocrinol Metab 1996;81:3270-6.

78 Dolder CR NM. Hypnosedative-induced complex behaviours : incidence, mechanisms and management. CNS Drugs 2008;22:1021-36.

79 Silverman D, Gendreau M. Medical issues associated with commercial flights. The Lancet 2009;373:2067-77.10.1016/S0140-6736(09)60209-9
80 Burke TM, Markwald RR, McHill AW, et al. Effects of caffeine on the human circadian clock in vivo and in vitro. Sci Trans/ Med 2015;7:146.

81 WADA. [Internet] World Anti-Doping Agency. Prohibited list. Montreal, 2017. Available: https://www.wada-ama.org/en/what-we-do/prohibited-list

82 Dickmeis T. Glucocorticoids and the circadian clock. J Endocrinol 2009:200:3-22.10.1677/JOE-08-0415

83 Frew AJ. Glucocorticoids. Clin Immunol: Elsevier 2019:e1161.

84 Cuesta M, Cermakian N, Boivin DB. Glucocorticoids entrain molecular clock components in human peripheral cells. Faseb J 2015;29:1360-70.

85 Roth T, Roehrs T, Koshorek G, et al. Sedative effects of antihistamines. J Allergy Clin Immunol 1987;80:94-8.

86 Richardson GS, Roehrs TA, Rosenthal L, et al. Tolerance to daytime sedative effects of H1 antihistamines. J Clin Psychopharmacol 2002;22:511-5.

87 Manning C, Scandale L, Manning EJ, et al. Central nervous system effects of meclizine and dimenhydrinate: evidence of acute tolerance to antihistamines. J Clin Pharmacol 1992;32:996-1002.10.1002/j.1552-4604.1992.tb03801.x 